\title{
On zeros of the characteristic determinant of the spectral problem for a third-order differential operator on a segment with nonlocal boundary conditions
}

Nurlan S Imanbaev ${ }^{1 *}$, Baltabek E Kanguzhin ${ }^{2}$ and Burkhan T Kalimbetov ${ }^{1}$

${ }^{*}$ Correspondence:

imanbaevnur@mail.ru

1 Department of Mathematics,

A. Yasawi International

Kazakh-Turkish University,

Turkestan, Kazakhstan

Full list of author information is

available at the end of the article

\begin{abstract}
In the paper, we consider distribution of eigenvalues of a third-order differential operator with nonlocal boundary conditions on a segment. The characteristic operator of the considered spectral problem is an entire function that has an integral representation. Countability and asymptotics of zeros for an entire function on the complex plane in sectors with a small span along the imaginary axis is proved.
\end{abstract}

\section{Introduction}

We consider the question of zeros distribution for entire functions of the form

$$
f(z)=1+A z+z^{2} \cdot \iint_{[0, b] \times[0, b]} \sum_{j=0}^{2} \exp \left(\rho\left(\omega_{j} x+\omega_{j+1} y\right)\right) \cdot D(x, y) d x d y
$$

here $\rho=\sqrt[3]{z}, \omega_{j}=\exp (2 \pi i j / 3), i=\sqrt{-1}, A$ is a constant, $D(x, y)$ is an absolutely integrated function.

The problems on eigenvalues for some classes of differential operators are reduced to a similar problem. In particular, the following problem on eigenvalues reduces to the studied question:

$$
\begin{aligned}
& y^{\prime \prime \prime}(x)+P_{1}(x) y^{\prime}(x)+P_{0}(x) y(x)=\lambda y(x), \quad x \in(0 ; b), \\
& y(0)=\lambda \int_{0}^{b} y(x) \sigma_{1}(x) d x, \\
& y^{\prime}(0)=\lambda \int_{0}^{b} y(x) \sigma_{2}(x) d x, \\
& y^{\prime \prime}(0)=\lambda \int_{0}^{b} y(x) \sigma_{3}(x) d x,
\end{aligned}
$$

where $\sigma_{1}(x), \sigma_{2}(x), \sigma_{3}(x) \in L_{2}(0 ; b), P_{1}(x), P_{0}(x) \in C^{\prime}(0 ; b)$, everywhere solvable in $L_{2}(0 ; b)$ multipoint problems are contained among them.

(c) 2013 Imanbaev et al.; licensee Springer. This is an Open Access article distributed under the terms of the Creative Commons Attribution License (http://creativecommons.org/licenses/by/2.0), which permits unrestricted use, distribution, and reproduction in any medium, provided the original work is properly cited. 
Works [1-6] are devoted to study of zeros of entire functions having integral representations.

Sometimes entire functions of the form (1) are the same as the quasipolynomials, the zeros of which are studied in works [7-9]. Connection between zeros of quasipolynomials and spectral problems is reflected in $[10,11]$.

The following theorems take place.

Theorem 1 Let $D(x, y)$ be an absolutely integrated on the square $[0, b] \times[0, b]$ function. If $D(x, y)$ is continuous and different from zero in a neighborhood of the square, then zeros of the function $f(z)$, which are sufficiently large by the modulus, can be located only in the sectors of an arbitrarily small span

$$
\left|\operatorname{Arg} z \pm \frac{\pi}{2}\right|<\varepsilon
$$

According to results of the monograph [11], there are exactly six critical rays on the plane $\rho$, i.e., $\arg \rho=\frac{\pi}{6}+\frac{\pi k}{3}, k=0,1,2,3,4,5$.

Theorem 2 Let conditions of Theorem 1 be realized, and let the value $\omega_{1} D(b, 0)+D(0, b)$ is different from zero. Then zeros of the function $f(z)$, which are sufficiently large by the modulus and are belonged to the sector $\left|\arg z-\frac{\pi}{2}\right|<\varepsilon$, have the asymptotics

$$
\begin{aligned}
\sqrt[3]{z_{k}}= & \left(\frac{-2 i k \pi}{b \omega_{2}}+\frac{1}{b \omega_{2}} \ln \frac{\omega_{1} D(b, 0)+D(0, b)}{D(b, b)}\right) \\
& +\underline{\underline{O}}\left(\max \left(\ln k \omega\left(\sqrt{2} \frac{\ln k}{k}\right) ; 1\right)\right), \quad k \geq 1,
\end{aligned}
$$

where $\omega(\delta)$ is the continuity modulus of the function $D(x, y)$.

Theorem 3 Let conditions of Theorem 1 be realized, and let the value $D(b, 0)+\omega_{2} D(0, b)$ be different from zero. Then the zeros of the function $f(z)$, which are sufficiently large by the modulus and are belonged to the sector $\left|\arg z+\frac{\pi}{2}\right|<\varepsilon$, have the asymptotics

$$
\begin{aligned}
\sqrt[3]{z_{k}}= & \left(\frac{-2 i k \pi}{b \omega_{1}}+\frac{1}{b \omega_{1}} \ln \frac{\omega_{1} D(b, 0)+\omega_{2} D(0, b)}{D(b, b)}\right) \\
& +\underline{\underline{O}}\left(\max \left(\ln k \omega\left(\sqrt{2} \frac{\ln k}{k}\right) ; 1\right)\right), \quad k \geq 1,
\end{aligned}
$$

where $\omega(\delta)$ is the continuity modulus of the function $D(x, y)$.

Theorems 1, 2, 3 are proved in [12]. In the present paper, we prove the following.

\section{Main results}

Theorem 4 Let the function $f(z)$ have the form (1). Suppose that the function $D(x, y)$ has continuous partial derivatives up to the third order by variables $x$ and $y$ in the square $[0, b] \times[0, b]$. If $\omega_{1} D(b, 0)+D(0, b) \neq 0, D(b, b) \neq 0$, then the zeros $z_{k}$ of the function $f(z)$ have the same asymptotics as in Theorems 2 and 3 ; the remainder term of which is written in the form of $\underline{\underline{O}}\left(\frac{1}{k}\right)$. 
Numbers, expressed by logarithms in Theorems 2 and 3, can be included in the remainder terms. At the same time, these values make the asymptotics more precise essentially in Theorem 4.

Proof Let $\rho$ be changed along the ray $\rho=|\rho| \exp (i \psi)$, where $\psi$ is a constant. Choose the point $\left(x_{0}, y_{0}\right)$ and the index $j_{0}$ from the condition

$$
\max _{0 \leq j \leq 2} \max _{(x, y) \in[0, b]^{2}} \operatorname{Re} \rho\left(\omega_{j} x+\omega_{j+1} y\right)=\operatorname{Re} \rho\left(\omega_{j 0} x_{0}+\omega_{j 0+1} y_{0}\right) .
$$

Consider a critical ray $\arg \rho=\frac{\pi}{6}$. Then

$$
\max _{0 \leq j \leq 2} \max _{(x, y) \in[0, b]^{2}} \operatorname{Re} \rho\left(\omega_{j} x+\omega_{j+1} y\right)=\operatorname{Re} \rho\left(\omega_{j 0} x_{0}+\omega_{j 0+1} y_{0}\right)
$$

is attained on a side of the square $[0, b] \times[0, b]$ at $j_{0}=2$.

Integration by parts of $f(z)$ gets the relations

$$
\begin{aligned}
f(z)= & +A z+z^{2} \sum_{j=0}^{2}\left[-\frac{D(b, b)}{\rho^{2} \omega_{j} \omega_{j+1}} \exp \left(\rho\left(\omega_{j} b+\omega_{j+1} b\right)\right)\right. \\
& -\frac{D(0, b)}{\rho^{2} \omega_{j} \omega_{j+1}} \exp \left(\rho \omega_{j+1} b\right)-\frac{D(b, 0)}{\rho^{2} \omega_{j} \omega_{j+1}} \exp \left(\rho \omega_{j} b\right)+\frac{D(0,0)}{\rho^{2} \omega_{j} \omega_{j+1}} \\
& -\int_{0}^{b} \frac{\partial D(x, b)}{\partial x} \cdot \frac{1}{\rho^{2} \omega_{j} \omega_{j+1}} \cdot \exp \left(\rho\left(\omega_{j} x+\omega_{j+1} b\right)\right) d x \\
& +\int_{0}^{b} \frac{\partial D(x, 0)}{\partial x} \cdot \frac{1}{\rho^{2} \omega_{j} \omega_{j+1}} \cdot \exp \left(\rho \omega_{j} x\right) d x-\int_{0}^{b} \frac{1}{\rho^{2} \omega_{j} \omega_{j+1}} \frac{\partial D(b, y)}{\partial y} \\
& \times \exp \left(\rho\left(\omega_{j} b+\omega_{j+1} y\right)\right) d y+\int_{0}^{b} \frac{1}{\rho^{2} \omega_{j} \omega_{j+1}} \cdot \frac{\partial D(0, y)}{\partial y} \cdot \exp \left(\rho \omega_{j+1} y\right) d y \\
& \left.+\frac{1}{\rho^{2} \omega_{j} \omega_{j+1}} \int_{0}^{b} d x \int_{0}^{b} \frac{\partial D(x, y)}{\partial x \partial y} \cdot \exp \left(\rho\left(\omega_{j} x+\omega_{j+1} y\right)\right) d y\right] .
\end{aligned}
$$

Introduce the function

$$
h(z)=\frac{z^{2} D(b, b)}{\rho^{2} \omega_{2} \omega_{0}} \cdot \exp \left(\rho\left(\omega_{2} b+\omega_{0} b\right)\right)-\frac{z^{2}}{\rho^{2}}\left(\frac{D(0, b)}{\omega_{2} \omega_{0}}+\frac{D(b, 0)}{\omega_{0} \omega_{1}}\right) \cdot \exp \left(\rho \omega_{0} b\right)
$$

and also the difference $G(z)=f(z)-h(z)$. The zeros of the function $h(z)$ are written out as follows:

$$
\rho_{k}^{0}=\frac{-2 \pi k i}{b \omega_{2}}+\frac{1}{b \omega_{2}} \ln \frac{\omega_{1} D(b, 0)+D(0, b)}{D(b, b)}, \quad k \geq 1 .
$$

Consider the square $T$ with the side $2 \varepsilon$ and the center situated in the point $\rho_{k}^{0} \omega_{2} b$ on the complex plane $\rho b \omega_{2}$. Choose $\varepsilon$ such that conditions of the Rushe theorem are realized, and $\varepsilon$ could be minimal from possible ones. Let us show feasibility of conditions of the Rushe theorem $|G(z)|<|h(z)|$ on the sides of the square $T$. In this order, we compare the majorant of the function $G(z)$ and the minorant of the function $h(z)$, i.e., we should to verify that $\max _{T}|G(z)|<\min _{T}|h(z)|$. 
Estimate from below the function $h(z)$. Then we get the inequality

$$
|h(z)| \geq \frac{|z|^{2}}{|\rho|^{2}} \exp \left(\operatorname{Re} \rho \omega_{0} b\right) \cdot\left|\omega_{1} D(b, 0)+D(0, b)\right| \cdot \min \{\operatorname{tg} \varepsilon ; 1-\exp (-\varepsilon)\} .
$$

The part of summands, entering into the function $G(z)$, includes exponents, the real numbers of indexes of which are directly less than one of numbers $\operatorname{Re} \rho \omega_{0} b$ and $\operatorname{Re} \rho\left(\omega_{2} b+\right.$ $\left.\omega_{0} b\right)$. Real parts of the indexes for each of these summands can be estimated by

$$
\left\{\operatorname{Re} \rho \omega_{0} b ; e \rho\left(\omega_{2} b+\omega_{0} b\right)\right\}
$$

The remaining summands of the integral containing exponents with real numbers of the indexes are equal to one from the numbers $\operatorname{Re} \rho \omega_{0} b$ and $\operatorname{Re} \rho\left(\omega_{2} b+\omega_{0} b\right)$. Using the smoothness properties of the function $D(x, y)$, we integrate by parts each of these integrals. Then we estimate them from above. As a result, we get the inequality

$$
\begin{aligned}
|G(z)| \leq & \frac{2|z|^{2}}{|\rho|^{2}} \exp \left(\operatorname{Re} \rho \omega_{0} b\right) \cdot\left(1+\exp \left(\operatorname{Re} \rho \omega_{2} b\right)\right) \\
& \times\left\{\exp \left(\rho \omega_{1} b\right) \cdot|D(b, b)|+(|D(0, b)|+|D(b, 0)|) \cdot \exp \left(\operatorname{Re} \rho\left(\omega_{2}-\omega_{0}\right) b\right)\right. \\
& +\frac{3}{2} \times \exp \left(-\operatorname{Re} \rho \omega_{0} b\right) \cdot|D(0,0)|+\frac{3}{2} \frac{|\rho|^{2}}{|z|^{2}} \cdot\left[\left|\frac{\partial D(b, b)}{\partial x}\right|+\left|\frac{\partial D(0, b)}{\partial x}\right|\right. \\
& +\int_{0}^{b}\left|\frac{\partial^{2} D(x, b)}{\partial x^{2}}\right| d x+\left|\frac{\partial D(b, 0)}{\partial x}\right|+\left|\frac{\partial D(0,0)}{\partial x}\right| \\
& +\int_{0}^{b}\left|\frac{\partial^{2} D(x, 0)}{\partial x^{2}}\right| d x+\left|\frac{\partial D(b, b)}{\partial y}\right|+\left|\frac{\partial D(b, 0)}{\partial y}\right| \\
& +\int_{0}^{b}\left|\frac{\partial^{2} D(b, y)}{\partial y^{2}}\right| d y+\left|\frac{\partial D(0, b)}{\partial y}\right|+\left|\frac{\partial D(0,0)}{\partial y}\right| \\
& +\int_{0}^{b}\left|\frac{\partial^{2} D(0, y)}{\partial y^{2}}\right| d y+\int_{0}^{b}\left|\frac{\partial^{2} D(x, b)}{\partial x \partial y}\right| d x \\
& \left.\left.+\int_{0}^{b}\left|\frac{\partial^{2} D(x, 0)}{\partial x \partial y}\right| d x+\int_{0}^{b} \int_{0}^{h}\left|\frac{\partial^{3} D(x, y)}{\partial x \partial y^{2}}\right| d y d x\right]\right\} .
\end{aligned}
$$

Now choose $\varepsilon>0$ such that the following inequality holds on the sides of the square $T$ :

$$
\begin{aligned}
& \frac{|z|^{2}}{|\rho|^{2}} \exp \left(\operatorname{Re} \rho \omega_{0} b\right) \cdot\left|\omega_{1} D(b, 0)+D(0, b)\right| \cdot \min \{\operatorname{tg} \varepsilon ; 1-\exp (-\varepsilon)\} \\
& \quad \geq \text { the right side of the inequality (4). }
\end{aligned}
$$

Then we obtain from here an inequality for choosing $\varepsilon$ :

$$
\begin{aligned}
& \left|\omega_{1} D(b, 0)+D(0, b)\right| \cdot \min \{\operatorname{tg} \varepsilon ; 1-\exp (-\varepsilon)\} \\
& \geq 2\left(1+\exp \left(\operatorname{Re} \rho \omega_{2} b\right)\right) \\
& \quad \times\left\{\exp \left(\operatorname{Re} \rho \omega_{1} b\right) \cdot|D(b, b)|+(|D(0, b)|+|D(b, 0)|) \cdot \exp \left(\operatorname{Re} \rho\left(\omega_{2}-\omega_{0}\right) b\right)\right. \\
& \left.\quad+\frac{3}{2} \exp \left(-\operatorname{Re} \rho \omega_{0} b\right) \cdot|D(0,0)|+\frac{3}{2} \frac{|\rho|^{2}}{|z|^{2}} B\right\}
\end{aligned}
$$


where indexes of the following exponents $\exp \left(\operatorname{Re} \rho \omega_{1} b\right),\left(\operatorname{Re} \rho\left(\omega_{2}-\omega_{0}\right) b\right), \exp \left(-\operatorname{Re} \rho \omega_{0} b\right)$ are negative. For the best estimate, take the most minimal from indexes of these exponents. For this, introduce the notation:

$$
-\operatorname{Re} \rho b \delta=\max \left\{\operatorname{Re} \rho b \omega_{1}, \operatorname{Re} \rho b\left(\omega_{2}-\omega_{0}\right),-\operatorname{Re} \rho b \omega_{0}\right\},
$$

where $\delta>0$. Because the value $\rho$ is equivalent to $\frac{2 i \pi k}{b \omega_{2}}$ on the indicated square $T$, we have

$$
\begin{aligned}
2(1+ & \exp (\operatorname{Re} 2 i \pi k)) \times\{\exp (-\operatorname{Re} \rho b \delta) \cdot|D(b, b)|+(|D(0, b)|+|D(b, 0)|) \\
& \left.\times \exp (-\operatorname{Re} \rho b \delta)+\frac{3}{2} \exp (-\operatorname{Re} \rho b \delta) \cdot|D(0,0)|+\frac{3}{4 \pi k} B\right\} \\
\geq & \left|\omega_{1} D(b, 0)+D(0, b)\right| \times \min \{\operatorname{tg} \varepsilon ; 1-\exp (-\varepsilon)\} .
\end{aligned}
$$

Denote by $A=\left|\omega_{1} D(b, 0)+D(0, b)\right|$. Then we obtain

$$
4 \exp (-\operatorname{Re} \rho b \delta) \cdot C+\frac{3}{4 \pi k}=A \varepsilon \text {. }
$$

Since $(-\operatorname{Re} \rho b \delta)$ is negative, we have $\varepsilon \approx \underline{\underline{Q}}\left(\frac{1}{k}\right)$. So, Theorem 4 is proved.

Remark Results of Theorems 1-3 are correct for the case of $D(x, y)$ is continuous in a neighborhood of corners of the square $[0, b] \times[0, b]$.

\section{Competing interests}

The authors declare that they have no competing interests.

\section{Authors' contributions}

The authors have made the same contribution. All authors read and approved the final manuscript.

\section{Author details}

'Department of Mathematics, A. Yasawi International Kazakh-Turkish University, Turkestan, Kazakhstan. ${ }^{2}$ Department of Fundamental mathematics, Al-Farabi Kazakh National University, Almaty, Kazakhstan.

Received: 28 January 2013 Accepted: 4 April 2013 Published: 18 April 2013

\section{References}

1. Titchmarsh, EC: The zeros of certain integral functions. Proc. Lond. Math. Soc. s2-25, 283-302 (1926)

2. Polya, G: Uber die Nullstelellen gewisser ganzer Funktionen. Math. Z. 2, 352-383 (1918)

3. Gartwright, ML: The zeros of certain integral functions. Q. J. Math. 1(1), 38-59 (1930)

4. Sedletskiy, AM: On zeros of the Fourier transform of the finite measure. Mat. Zametki 53(1), 111-119 (1993)

5. Anderson, DR, Ma, R: Second-order $n$-point eigenvalue problems on time scales. Adv. Differ. Equ. 2006, Article ID $59572(2006)$

6. Gao, J, Sun, D, Zhang, M: Structure of eigenvalues of multi-point boundary value problems. Adv. Differ. Equ. 2010, Article ID 381932 (2010)

7. Bellman, R, Cooke, K: Difference-Differential Equations, p. 548. Mir, Moscow (1967)

8. Leontyev, AF: Integral Functions and Series of Exponents, p. 176. Moscow (1983)

9. Hald, OH: Discontinuous inverse eigen value problems. Commun. Pure Appl. Math. 37, $539-577$ (1984)

10. Naymark, MA: Linear Differential Operators, p. 496. Nauka, Moscow (1969)

11. Shkalikov, AA: On baseness of eigenfunctions of ordinary differential operators with integral boundary conditions. Vestn. MGU, Ser. Mat. Mech. 6, $12-21$ (1982)

12. Imanbaev, NS, Kanguzhin, BE: On zeros of entire functions that have an integral representation. Izv. Nats. Akad. Nauk Kazakhstan, Ser. Fiz.-Mat. 3, 47-51 (1995) 